\title{
Sequential optimization of strip bending process using Multiquadric Radial Basis Function surrogate models
}

\author{
Jos Havinga ${ }^{1, a}$, Ton van den Boogaard ${ }^{1, b}$ and Gerrit Klaseboer ${ }^{2, c}$ \\ ${ }^{1}$ University of Twente, Nonlinear Solid Mechanics \\ PO Box 217, 7500AE, Enschede, The Netherlands \\ ${ }^{2}$ Philips Consumer Lifestyle, PO Box 201, 9200AE, Drachten, The Netherlands \\ a G.T.Havinga@utwente.nl, b A.H.vandenBoogaard@utwente.nl, ${ }^{\mathrm{c}}$ Gerrit.Klaseboer@Philips.com
}

\begin{abstract}
Keywords: Strip bending, Surrogate modeling, Sequential Robust Optimization, Multiquadric Radial Basis Functions, Robust Expected Improvement
\end{abstract}

\begin{abstract}
Surrogate models are used within the sequential optimization strategy for forming processes. A sequential improvement (SI) scheme is used to refine the surrogate model in the optimal region. One of the popular surrogate modeling methods for SI is Kriging. However, the global response of Kriging models deteriorates in some cases due to local model refinement within SI. This may be problematic for multimodal optimization problems and for other applications where correct prediction of the global response is needed. In this paper the deteriorating global behavior of the Kriging surrogate modeling technique is shown for a model of a strip bending process. It is shown that a Radial Basis Function (RBF) surrogate model with Multiquadric (MQ) basis functions performs equally well in terms of optimization efficiency and better in terms of global predictive accuracy. The local point density is taken into account in the model formulation.
\end{abstract}

\section{Introduction}

Nowadays it is good practice to use Finite Element (FE) models for the design of production processes, such as the one shown in Fig. 1. A designer may base several design choices on the results of a FE model. Optimization schemes may be used to automate the search for the optimal design. Robust Optimization methods can be used to take into account process variations (e.g. variations of material properties) which may cause variations to the process objective (e.g. product geometry) or to the process constraints (e.g. maximum process force).

The optimization procedure may need a large number of process evaluations. It would usually be too costly to perform these evaluations directly on the FE models. Hence a so-called surrogate model may be used to predict the FE response at any point in the combined design-noise space. This surrogate-model interpolates a set of points which have been evaluated by the FE model. The set of points that is used to construct the surrogate model may be expanded during the optimization procedure, increasing the surrogate model accuracy in the optimal region. This may be achieved within a sequential improvement scheme [1].

The surrogate model is an important building block of the sequential optimization strategy. A prediction of the surrogate model error is required within this strategy. Kriging surrogate models are popular for sequential optimization since they

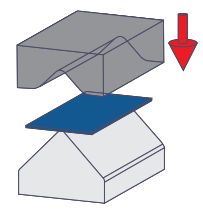

Figure 1: Bending of a strip process. include a prediction error by definition. However, Kriging originated in the field of geostatistics [2] and some assumptions of the method may be called in question when applying it to deterministic FE models. Moreover, deteriorating accuracy of the model is observed when a large number of points is sampled in a region of specific interest. This does not have to be problematic since the accuracy in the region of interest does not deteriorate. Hence, the model optimum may still be found efficiently using Kriging. However, worsening of the global response of the surrogate model may become a problem in multimodal optimization problems, since the risk exists that the algorithm 
focusses on one region, deteriorating the response of another region where the optimum might be found.

Furthermore, other applications may also need accurate global prediction when the density of observations is increased locally. An example can be found in the MEGaFiT [3] project where this research is part of. Within the MEGaFiT project it is aimed to control a forming production process using a feedback control system during production. The goal is to construct a control strategy which is based on process knowledge gained through FE modeling of the process. Surrogate models must be constructed to define the feedback behavior. It is reasonable to define regions of higher sampling of the feedback surrogate models, for example based on the local non-linearity of the model. However, it is essential that the global response of the surrogate model is reasonably accurate, since it is not known a priori which region of the surrogate model is going to be used by the control system.

Based on the above mentioned reasons a different surrogate modeling technique is proposed for sequential optimization in this paper. Radial Basis Functions (RBF) with Multiquadric (MQ) basis can be used within the sequential optimization strategy if some assumptions on the modeling error are made. In a famous comparative study performed by Franke [4] good predictive accuracy of the MQ RBF is found. In the current study MQ RBF proves to be well applicable to the sequential improvement robust optimization strategy.

Another important building block of the sequential optimization strategy is a criterion to select new infill points during the sequential optimization iterations. For deterministic optimization the Expected Improvement (EI) proved to be an efficient criterion to extend the DoE [5]. For robust optimization the selection of an infill point is less straightforward, since it makes no sense to search for an optimal value in the noise dimension. Some proposals for a criterion are found in literature [6,7], however we propose a Robust Expected Improvement (REI) criterion which has a closer link to the deterministic version of the EI.

The sequential optimization scheme will be applied to the bending of a strip. The process is shown in Fig. 1. The goal is to find an optimal punch displacement while taking yield stress and plate thickness variation into account. Although simple at first glance, bending of a strip is a complicated process due to the multiple contact areas which increase the non-linearity of the problem. In this study the optimization problem is even multimodal. It is shown that the MQ RBF surrogate model provides good results within the sequential optimization scheme.

This paper is structured as follows: first the surrogate modeling techniques used in this research are discussed. The sequential optimization scheme including infill criterion is then treated, followed by a presentation of the model of the strip bending process. The optimization results are shown thereafter and the article is closed with some concluding remarks.

\section{Surrogate modeling}

As stated above, the surrogate modeling technique chosen within a sequential optimization scheme may influence the results strongly in terms of accuracy and efficiency. A surrogate model $\hat{f}(x)$ is an approximation of an unknown function $f(x)$. A set of observations $y^{(i)}$ of the unknown function at the DoE locations $x^{(i)}$ is used to construct the surrogate model. For interpolative methods the following holds:

$$
\hat{f}\left(x^{(i)}\right)=f\left(x^{(i)}\right) \equiv y^{(i)} \quad i=1 . . n .
$$

Kriging. The Kriging method is very popular for sequential optimization purposes since it includes a prediction error by definition: the observations $y^{(i)}$ are seen as a realization of a stochastic process. An expected value and a mean square error may be computed at any point within the domain of the model, based on some assumptions about the spatial correlation of the stochastic process. In Universal Kriging, a trend is extracted from the data and the error with regard to the trend is seen as the stochastic process which is interpolated. Typically, a lower order polynomial is used to describe 
the trend. A common choice for the correlation function is a Gaussian function. It is assumed that the amount of spatial correlation may be different per dimension. Solving this statistical problem leads to the following solution:

$$
\hat{f}\left(x^{*}\right)=\sum_{k=0}^{p} \beta_{k} f_{k}\left(x^{*}\right)+\sum_{i=1}^{n} \lambda_{n} \exp \left(-\sum_{j=1}^{d} \theta_{j}\left|x_{j}^{(i)}-x_{j}^{*}\right|^{2}\right) .
$$

The first part of Eq. 2 equals the polynomial trend, the second part equals the sum over a set of Gaussian basis functions placed at the $n$ observation points. The spatial correlation parameters $\theta_{j}$ must be determined for all $d$ dimensions. Usually, a likelihood function is used to find the correlation parameters $\theta_{j}$ that maximize the likelihood of the given set of observations.

Despite of Kriging being a surrogate modeling technique for stochastic processes, good results can be achieved when using Kriging to fit deterministic FE models [1]. However, it may be questioned how the assumptions of the Kriging method influence the prediction of a FE model. It is for example not obvious that the dimensional correlation parameters $\theta_{j}$ are constant within the whole domain, as assumed within Kriging. Above that, Kriging has trouble with DoE's which are not uniformly distributed in space. During sequential optimization, points are added to the model in the region of interest. This normally leads to a decreasing size of the spatial correlation parameters $\theta_{j}$, leading the Gaussian basis functions to become narrow. This may cause the global response of the Kriging surrogate model to worsen. This is problematic for multimodal optimization problems and other applications such as for the production control strategy within the MEGaFiT project.

Radial Basis Functions. Based on the preceding reasoning, our attempt is to select a surrogate modeling technique which performs well for DoE's which have a varying point density throughout the domain. The aim is to compare the behavior of this model to the behavior of Kriging models in a sequential optimization scheme.

RBF surrogate models are similar to Kriging models in the sense that the model consists of a sum of basis functions placed within the domain, optionally combined with a polynomial fit. However, Kriging is usually constrained to basis functions which are decaying from the center point, since the shape of the basis function is determined by the shape of the assumed spatial correlation function. In contrary, RBF can be computed with all types of basis functions. Several variations have been proposed and used within literature: gaussian, thin plate spline, multiquadric, inverse multiquadric and other functions [8].

In this study, Multiquadric (MQ) basis functions have been selected due to their high predictive quality obtained within some studies [4]. Having $n$ basis functions at $n$ DoE points, the formulation of the MQ RBF is as follows:

$$
\hat{f}\left(x^{*}\right)=\frac{1}{n} \sum_{i=1}^{n} y^{(i)}+\sum_{i=1}^{n} \lambda_{i} \sqrt{\left\|x^{(i)}-x^{*}\right\|^{2}+\theta_{i}^{2}}
$$

Given a certain set of shape parameters $\theta_{1 . . n}$, the $n$ unknown weights $\lambda_{i}$ can easily be determined using Eq. 1: the number of unknowns $\lambda_{i}$ is equal to the amount of equations. The remaining question is how to determine the shape parameters $\theta_{1 . . n}$. A common way to do this is to use a constant shape parameter at each basis $\left(\theta_{1 . . n}=\theta_{\text {global }}\right)$ and then determine the global shape parameter $\theta_{\text {global }}$ using the Leave-One-Out-Cross-Validation (LOOCV) measure. The LOOCV measure is:

$$
\operatorname{LOOCV}\left(\theta_{\text {global }}\right)=\left(\sum_{i=1}^{n}\left(\hat{f}\left(x^{(i)}\right)-\hat{f}^{(i)}\left(x^{(i)}\right)\right)^{2}\right)^{1 / 2}
$$

In Eq. 4, $\hat{f}^{(i)}(x)$ is the surrogate model constructed with all DoE points except point $x^{(i)}$. Shmuel Rippa showed that this measure can be computed at $O\left(n^{3}\right)$ instead of $O\left(n^{4}\right)$ cost [9]. Hence, 
an optimal fit may be found by selecting the shape parameter $\theta_{\text {global }}$ which minimizes the LOOCV measure.

The shape parameter of the MQ RBF influences the local behavior of the surrogate model (Fig. 2 ). The fit could be improved by varying the value of the shape parameter $\theta_{i}$ at each basis. However, it would be too expensive to include $n$ shape parameters $\theta_{1 . . n}$ in the LOOCV minimization scheme. Some authors propose to couple the local shape parameter $\theta_{i}$ to the distance to the nearest neighbor. This approach is adopted in this study, adapting the behavior of the surrogate model to the local point density. Hence, the local shape parameter $\theta_{i}$ is coupled to $d_{i}$, which is the distance between point $x^{(i)}$ and its nearest neighbor:

$$
\theta_{i}=\theta_{\text {global }} d_{i}
$$

Within the sequential optimization scheme, the prediction uncertainty of the surrogate model is needed to define the new infill point. In contrary to Kriging, prediction uncertainty is not included in the RBF formulation. However, the LOOCV measure is obtained during determination of the optimal shape parameter $\theta_{\text {global }}$. This measure can be used to compute a prediction uncertainty of the RBF model. A few assumptions on the prediction uncertainty have to be made.

Firstly, it is assumed that the model uncertainty at a location far from the DoE points is a normal distribution with standard deviation

$\sigma_{\max }$. Secondly, it is assumed that the correlation between two points is determined by the distance between these points. Hence, an untried

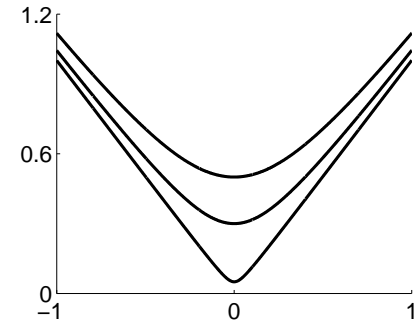

Figure 2: Effect of changing $M Q R B F$ shape parameter $\theta$ on basis function shape. point close to a DoE point will have a large correlation with that point and therefore the prediction uncertainty at this untried point will be small. Next, a shape for the correlation function must be assumed. In this study, a Gaussian function is chosen for the correlation function between the DoE point $x^{(i)}$ and the untried point $x^{*}$ :

$$
\rho_{i}\left(x^{*}\right)=e^{-\left(\frac{\left\|x^{(i)}-x^{*}\right\|}{2 \delta}\right)^{p}} .
$$

Given the correlations $\rho_{i}\left(x^{*}\right)$ with all DoE points and the standard deviation $\sigma_{\max }$, the prediction uncertainty at an untried point can be computed using a multivariate probability distribution.

The shape parameters $\delta$ and $p$ and the maximum standard deviation $\sigma_{\max }$ still have to be determined. It can be seen that these parameters must somehow correspond with the LOOCV measure. Given a model where one DoE point is left out, the uncertainty at this DoE location can be found for a given set of parameters $\delta, p$ and $\sigma_{\max }$. The known cross-validation error at this location is a realization of this uncertainty measure and the probability of this realization can be computed. Calculating and multiplying the probability of all cross-validation errors gives the likelihood of the set of cross-validation errors. As done with Kriging while computing the model parameters, it can be said that the likelihood of the model realization (cross-validation errors) for a given set of model parameters $\left(\delta, p\right.$ and $\left.\sigma_{\max }\right)$ is equal to the likelihood of the model parameters for a given model realization. Hence, the model parameters $\delta, p$ and $\sigma_{\max }$ can be found by maximizing the likelihood of the cross-validation values which have been found while fitting the RBF model.

By fulfilling this task, all ingredients needed to run a sequential optimization scheme are now found in the RBF model.

\section{Sequential Robust Optimization}

A robust optimum for the bending process must be found based on deterministic FE models. Given an assumption on the noise distribution influencing the process, Monte Carlo sampling can 
be performed at any design setting to compute the expected probability distribution of the process response. The optimization objective function may be written in terms of the mean and standard deviation found at a design point.

The optimization is performed on the surrogate model, which is not equal to the real process. During the optimization scheme, it is aimed to let the optimum on the surrogate model converge to the real optimum of the process ${ }^{1}$. This is done by successive addition of points to the surrogate model. In this way the accuracy of the prediction increases gradually.

A certain criterion for the selection of new points must be found. In deterministic optimization a straightforward criterion called Expected Improvement (EI) has been developed [5]. The reasoning is that the surrogate model gives a normal distributed prediction of the real function value $y^{*} \sim$ $N\left(\hat{y}, \sigma^{2}\right)$ at an untried point. Hence, there is a chance that the model at this location is better than the model at the best evaluated point $y^{(b e s t)}$ found up to that moment. The model improvement is defined as $y^{\text {(best) }}-y^{*}$ if $y^{(b e s t)}>y^{*}$ and zero otherwise ${ }^{2}$. Integration of the product of the improvement with the response probability distribution at an untried point gives the expected improvement. The point with the highest expected improvement is selected as the new point to be evaluated with the FE model.

Robust Expected Improvement. In the case of robust optimization the selection of a new point is more complicated. A few proposals are found in literature [6,7] but there has been little research on the efficiency of these criteria. In this study a criterion which has a close link to the deterministic EI criterion is proposed.

The objective function in the robust optimization scheme is defined in the design space, whereas the surrogate model prediction and uncertainty are given in the combined design and noise space. Hence there is no location in the design space where the objective function value is exactly known, since the noise space at any design point will always be finitely sampled. However, the current objective function value $\hat{Y}_{o b j}$ and the mean prediction error $\sigma^{2}$ in the noise direction can be computed at any design point. Hence, the objective function prediction at any point in the design space can be seen as a normal distribution: $Y_{o b j} \sim N\left(\hat{Y}_{o b j}, \sigma^{2}\right)$. The point with the best objective function value $Y_{o b j}{ }^{\text {(best) }}$ in the design space can be compared with any other objective function prediction in the design space $Y_{o b j}$. Note that the real value of both $Y_{o b j}{ }^{\text {(best) }}$ and $Y_{o b j}{ }^{*}$ will be a realization of a normal distribution. Hence, both normal distributions can be combined to an uncorrelated bivariate normal distribution. Within this space the improvement can be defined in the same way as done in the deterministic case. Finally, the determination of the Robust Expected Improvement (REI) is totally analogous to the deterministic case: the product of the improvement and the probability is integrated over the complete probability space. The only difference with the deterministic case is that the probability space is bivariate instead of univariate.

Using the REI a point in the design space can be selected for a new FE run. However, the location of this point in the noise space still has to be defined. At any point in the noise space the prediction uncertainty can be computed from the surrogate model. In his robust selection criterion, Jurecka proposed to select the point in noise space where the product between the point probability and the prediction uncertainty is the largest [6]. In this study the same criterion is adopted for the selection of the point in the noise dimension.

\section{Strip Bending Model}

The proposed robust sequential optimization scheme will be applied to the bending of a strip. The process is shown in Fig. 1. The geometry is shown in Fig. 3. The process is reasonably complicated due to the multiple moving contact areas on the punch and on the die side. Springback is calculated after the bending process. Two noise parameters are included in the optimization: the yield stress $\sigma_{y}$ and the strip thickness $t$. The final distance $D$ between the punch and the die is determined by minimizing the difference between the final strip angle $\theta$ and the target angle $90^{\circ}$ :

\footnotetext{
${ }^{1}$ Assuming that the FE model describes the real process.

${ }^{2}$ In the case that the optimization problem is written as a minimization of $y$.
} 
find $D$

$\min \left|\mu_{\theta}-90^{\circ}\right|+3 \sigma_{\theta}\left[^{\circ}\right]$

$0.4 \leq D \leq 0.65[\mathrm{~mm}]$

$\sigma_{y} \sim \mathrm{N}\left(350,6.66^{2}\right)[\mathrm{MPa}]$

$t \sim \mathrm{N}\left(0.51,0.01^{2}\right)[\mathrm{mm}]$

Within the sequential optimization scheme Monte Carlo sampling on the surrogate model is used to compute the mean angle $\mu_{\theta}$ and the standard deviation $\sigma_{\theta}$. The amount of points used in the Monte Carlo sampling is 2500 .

The surrogate modeling techniques used for the sequential optimization are Kriging with a $0^{\text {th }}$ order polynomial, Kriging with a $2^{\text {nd }}$ order polynomial and MQ RBF.

The initial DoE in the sequential optimization scheme is a

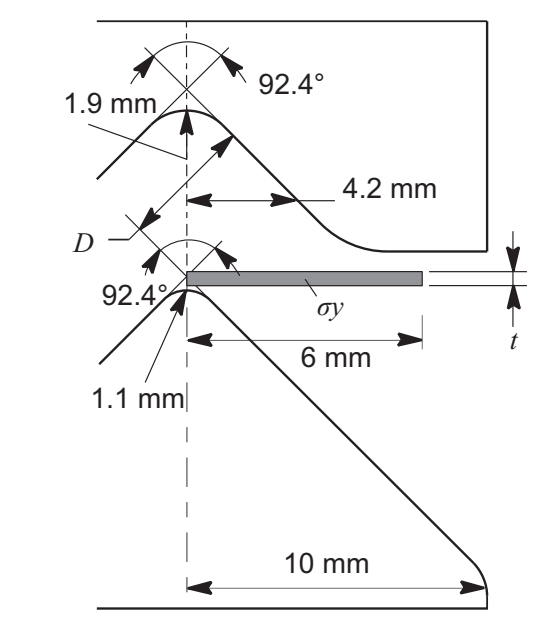

Figure 3: Tooling geometry and optimization parameters. latin hypercube with 12 points. For the noise parameters the interval of $\mu \pm 3 \sigma$ is used to construct the initial DoE.

The material is modeled with an elasto-plastic model. A Young's modulus $E$ of $210 \mathrm{GPa}$ is used. An experimental hardening curve is implemented in the FE model. Variation of the yield stress is modeled through a vertical shift of the hardening curve.

The implicit commercial code MSC Marc has been used to model the problem. Due to symmetry half of the sheet is modeled with 2D plane strain elements. The sheet is discretized using 500 quadrilateral elements over the strip width and 5 elements over the strip thickness. The elastic tools are modeled with a Young's modulus of $210 \mathrm{GPa}$. The runtime of one simulation is approximately seven minutes.

\section{Results}

Two local optima are observed in the robust optimization problem of the strip bending process. These local minima stand for two different ways to create a robust production process. The first optimum is the one where the final distance between punch and die $(D=0.502 \mathrm{~mm})$ is smaller than the mean strip thickness. Hence, the strip is flattened at the end of the punch stroke. The other solution $(D=0.528 \mathrm{~mm})$ is one where the final distance between punch and die is larger than the mean strip thickness. An extra set of model runs has been performed after the optimization to gain more knowledge on the exact behavior of the model at these design points. Monte Carlo sampling with 500 points has been executed on both locations. These results can be used to compare with the final predictions within the sequential optimization sequence.

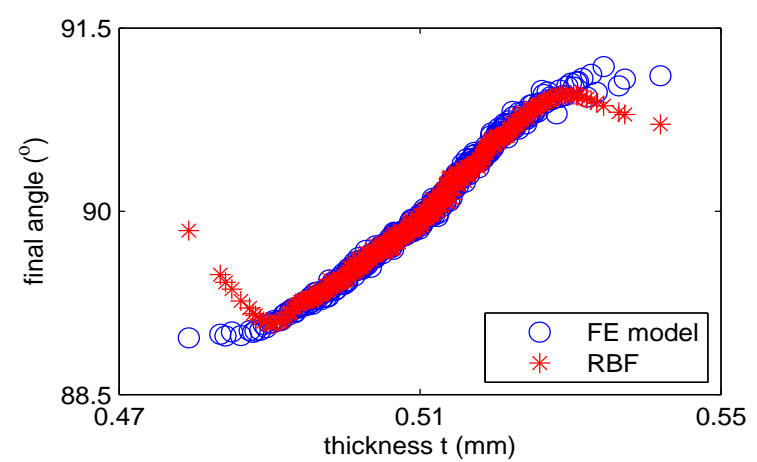

(a)

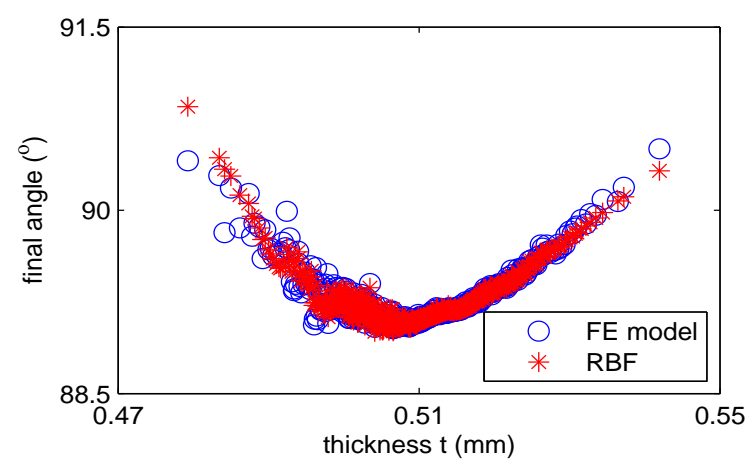

(b)

Figure 4: Response of the FE model (o) and the RBF model at the last (150 $\left.{ }^{\text {th }}\right)$ iteration (*) for both local optima. The response at $D=0.502 \mathrm{~mm}$ is shown on the left side (a) and the response at $D=$ 0.528 is shown on the right side (b). 
Fig. 4 shows the response of the FE model at the 500 Monte Carlo points for both optima. The 500 points have been evaluated on the final RBF surrogate model to compare the surrogate model results with the FE model results. It can be seen from Fig. 4 that the yield stress $\sigma_{y}$ distribution hardly affects the response of the process since the response is reasonably constant at any value of the thickness $t$. Furthermore, it can be seen that the final angle increases with increasing strip thickness $t$ in the region where $t$ is larger than the final punch displacement $D$. In this region the strip is flattened at the end of the punch stroke. However, for the case with final punch displacement $D=0.528 \mathrm{~mm}$ (Fig. 4b) it can be seen that the angle increases with decreasing thickness $t$ in the region where the thickness $t$ is smaller than the final punch displacement $D$. In this region the punch is not totally closed and the strip is not flattened.

An accurate objective function value $\left(\left|\mu_{\theta}-90^{\circ}\right|+3 \sigma_{\theta}\right)$ can be calculated at both local optima based on the $500 \mathrm{FE}$ calculations. For the first local optimum $(D=0.502 \mathrm{~mm})$ an objective function value of $1.55^{\circ}$ is found. The second local optimum $(D=0.528 \mathrm{~mm})$ has an objective function value of $1.42^{\circ}$. Thus, the global optimum of the optimization problem can be found at $D=0.528 \mathrm{~mm}$. The difference between both objective function values is small. Hence, it may be difficult for the sequential optimization algorithm to distinguish which of the local minima is the global minimum.

All evaluated surrogate modeling types manage to locate both optima within less than ten iterations. However, it takes many more iterations for the sequential optimization to get an accurate prediction of the complete noise domain of both optima, allowing to determine which local optimum is the global optimum. This is caused by the selection criterion of the new infill points in noise direction: locations with a higher probability to occur are preferred by the selection criterion. Hence, it takes a large amount of iterations for the criterion to select the points at the outer regions of the noise domain. It can be seen in Fig. 4a that the error of the RBF surrogate model at the outer region of the noise domain leads to an underprediction of the objective function value for $D=$ 0.502. For the Kriging models the same effect is observed, although less pronounced.

Global accuracy. Comparable results are found for the Kriging and RBF MQ surrogate models in terms of sequential robust optimization efficiency for this case of strip bending. However, it is stated in the introduction that the global accuracy of the surrogate modeling techniques may be of importance within a sequential optimization scheme and within other applications. Hence, the global predictive ability of the models has been evaluated. For this purpose, a DoE of 1000

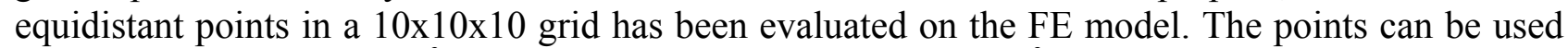
to get an estimate of the $R^{2}$ value of the different models. The $R^{2}$ value is a measure for the model accuracy and it is computed with Eq. 7. A $R^{2}$ value of 1 stands for a model which is able to exactly predict the response of the underlying model.

$$
R^{2}=1-\frac{\sum_{i=1}^{n}\left(y^{(i)}-\hat{f}\left(x^{(i)}\right)\right)^{2}}{\sum_{i=1}^{n}\left(y^{(i)}-\frac{1}{n} \sum_{i=1}^{n} y^{(i)}\right)^{2}} .
$$

For sequential robust optimization it makes no sense to continue adding points after the model converged to a stable prediction of the global optimum. However, we want to see the effect of increasing local point density on the global predictive ability of the methods. Therefore the sequential optimization is executed up to the addition of 150 points. Fig. 5 shows the average $R^{2}$ value of every set of ten consecutive surrogate models. It can be clearly observed that the global predictive ability of the $0^{\text {th }}$ order Kriging model deteriorates over time. However, the global predictive ability of the $2^{\text {nd }}$ order Kriging models and the RBF MQ models remains fairly constant over time. Hence, it can be seen that the choice of the surrogate modeling technique can have a large influence on the global predictive ability of the surrogate models in a sequential optimization scheme. In the case of the strip bending problem this did not noticeably influence the optimization efficiency. However, it can be reasoned that worsening of the global prediction may lead to a larger 
chance to miss a local optimum in a sequential optimization scheme when the algorithm focusses on one of the local optima.

\section{Conclusion}

A robust sequential optimization scheme has been used to find a robust process setting for a strip bending problem. A selection criterion for new points during the sequential optimization has been developed. The selection criterion proved to efficiently find new points to sample.

The strip bending problem has two different process settings which locally minimize the objective function. The global optimum of the optimization problem has been found.

Two different surrogate modeling techniques have been compared within the sequential robust optimization strategy. Both Kriging and MQ RBF located the optima within a reasonable amount of iterations. However, it has been observed that the global predictive ability of the $0^{\text {th }}$ order Kriging model deteriorates while adding points to the optimum regions. This may be problematic within more pronounced multimodal optimization problems and within other applications such as for the process control surrogate models developed within the MEGaFiT project.

\section{Acknowledgement}

The work leading to these results has received funding from the European Community's Seventh Framework Programme under grant agreement n FP7-285030.

\section{References}

[1] J.H. Wiebenga, A.H. van den Boogaard, G. Klaseboer, Sequential robust optimization of a Vbending process using numerical simulations, Struct. Multidisc. Optim., Vol. 46 (2012) 137-153.

[2] D.G. Krige, A statistical approach to some basic mine valuation problems on the Witwatersrand, J. S. Afr. I. Min. Metall., Vol. 52 No. 6 (2012) 119-139

[3] Information on www.megafit-project.eu

[4] R. Franke, Scattered data interpolation: tests of some methods, Math. Comput., Vol. 38 No. 157 (1982) 181-200

[5] D. Jones, M. Schonlau, W. Welch, Efficient Global Optimization of Expensive Black-Box Functions, J. Global Optim., Vol. 13 No. 4 (1998) 455-492

[6] F. Jurecka, Robust design optimization based on metamodeling techniques, $\mathrm{PhD}$ dissertation (1997)

[7] J. Janusevskis, R. Le Riche, Simultaneous kriging-based estimation and optimization of mean response, J. Global Optim., (2012) 1-24

[8] B. Fornberg, J. Zuev, The Runge phenomenon and spatially variable shape parameters in RBF interpolation, Comput. Math. Appl., Vol. 54 No. 3 (2007) 379-398

[9] S. Rippa, An algorithm for selecting a good value for the parameter $\mathrm{c}$ in radial basis function interpolation, Adv. Comput. Math., Vol. 11 No. 2 (1999) 193-210 